CHRISTIAN ZSCHIESCHANG

christian.zschieschang@serbski-institut.de

ORCID: 0000-0002-6698-6437

Serbski Institut / Sorbischen Institut

Cottbus, Deutschland
https://doi.org/10.17651/ONOMAST.65.1.3

Onomastica LXV, 2021

PL ISSN 0078-4648

\title{
PERSPEKTIVEN DER FLURNAMENFORSCHUNG IN DER SORBISCHEN LAUSITZ
}

Schlüsselwörter: Flurnamen, Namensammlungen, sorbisches Kulturerbe, Bürgerwissenschaft

In den langen Jahren meiner Tätigkeit am Leibniz-Insitut für Geschichte und Kultur des östlichen Europa (GWZO) ${ }^{1}$ hatte ich immer wieder mit Forschungsthemen zu tun, für die Publikationen von Barbara Czopek-Kopciuch von entscheidender Bedeutung waren ${ }^{2}$. In zwei Fällen bildeten sie sogar die wesentlichen Grundlagen von größeren Projekten, mit denen ich betraut war. Dies betraf Regionen an den entgegengesetzten Enden des polnischen Staatsgebietes: Zum einen ging es um die Namen von Mühlen, die eine spezifische und heterogene Namenklasse bilden und im Rahmen zweier interdisziplinärer Forschungsprojekte untersucht wurden ${ }^{3}$. Ein diesbezüglicher Aufsatz für das Gebiet der vormaligen Brandenburgischen Neumark (Rymar und Czopek, 1993) $^{4}$ ist hierbei in Umfang und Gründlichkeit der Bearbeitung geradezu vorbildlich für die gesamte mitteleuropäische Onomastik. Daneben bearbeitete ich in einem interdisziplinären Forschungskontext das Umfeld der im 10. Jahrhundert schriftlich bezeugten Čerwenischen Burgen. Diese werden seit mehr als zehn Jahren archäologisch intensiv untersucht ${ }^{5}$, worin

${ }^{1}$ Bis 2016 Geisteswissenschaftliches Zentrum für Geschichte und Kultur Ostmitteleuropas; das Akronym wurde als etablierte „Marke“ nach der Umbenennung beibehalten (vgl. https://www. leibniz-gwzo.de/de/institut/geschichte). Dort war ich beschäftigt bis Ende 2019; seit April $2020 \mathrm{am}$ Serbski Institut / Sorbischen Institut, Wótnožka za dolnoserbske slěźenja / Zweigstelle für niedersorbische Forschungen.

${ }^{2}$ Vgl. beispielsweise Zschieschang (2014) unter Rückgriff auf Czopek (1984).

${ }^{3}$ Usus aquarum. Mühlenbau, Wasser und Verkehr in der hochmittelalterlichen Kolonisation Ostmitteleuropas (2011-2013, gemeinsam mit Martina Maříková und Lucie Galusová); Usus aquarum. Mühlenbau, Wasser und Verkehr in der hochmittelalterlichen Kolonisation Ostmitteleuropas (II) Die Umgestaltung der Flusslandschaft beiderseits der Oder (2014-2019; gemeinsam mit Christoph Mielzarek). Dazu: Maříková und Zschieschang (2015); Mielzarek und Zschieschang (2019); Čīšangs [= Zschieschang], (2018); Zschieschang (2018).

${ }^{4}$ Zur Würdigung und Auswertung dieses Materials Zschieschang (2015a, S. 209-222).

${ }^{5}$ Beginnend mit dem Projekt Vergleichende Untersuchungen zum sozialen, wirtschaftlichen und kulturellen Wandel in den Grenz- und Kontaktzonen Ostmitteleuropas im Mittelalter (2008-2013); 
neben einer Vielzahl weiterer geistes- und naturwissenschaftlichen Disziplinen auch die Toponomastik eingebunden war (Zschieschang, 2017; 2019). Die wesentliche Arbeitsgrundlage hierfür, das Ortsnamenlexikon für große Teile dieser Region, stammte von Barbara Czopek-Kopciuch (Czopek, 1988). Diese Aufzählung aus einer externen Perspektive lässt erahnen, wie groß die Bedeutung der Forscherpersönlichkeit, der wir mit diesem Band gedenken, gewesen ist.

Einen Großteil ihres wissenschaftlichen Wirkens hat Barbara CzopekKopciuch den Siedlungsnamen gewidmet. Deren Bearbeitung für ein größeres Areal ist eine sehr aufwändige Angelegenheit. Noch komplexer ist die Sammlung und Analyse von Flurnamen zu bewältigen, die in der traditionellen Onomastik, bei der die historisch-etymologische Bearbeitung der Namen im Mittelpunkt steht ${ }^{6}$, der Aufarbeitung der Siedlungsnamen in der Regel folgt. Flurnamen liegen in ungeheuer großer Zahl vor und sind sehr heterogen. Sie sind in unterschiedlichen zeitlichen, sprachlichen, dialektalen und sozialen Kontexten entstanden. Daher ist es kaum möglich, „die“ Flurnamen in totaler Vollständigkeit zu sammeln und zu bearbeiten (Zschieschang, 2015b). Vielmehr kommt es darauf an, aus der Fülle des Materials die sprach- und kulturgeschichtlich relevanten Bildungen zu gewinnen.

Hierfür steht bekanntermaßen ein umfangreiches und vielfältiges Methodenspektrum zur Verfügung, dessen Breite derjenigen der thematische Zielstellungen der Flurnamenforschung sowie dem fallweise sehr unterschiedlichen Charakter der zur Verfügung stehenden Quellen entspricht. Für eine bestimmte Art und Weise der Flurnamenforschung zu plädieren, heißt nicht, die anderen Möglichkeiten geringzuschätzen. Hinsichtlich einer historischen, diachronen Untersuchung erscheint es besonders zielführend zu sein, Flurnamen im Rahmen der einzelnen Gemarkung bzw. der zu einer Siedlung gehörenden Flur zu betrachten. Deren Bewohner bildeten über Jahrhunderte hinweg eine relativ geschlossene Kommunikationsgemeinschaft. Insbesondere im Kontext der bis zum 19. Jahrhundert weit verbreiteten Dreifelderwirtschaft mit ihrem Flurzwang mussten die landwirtschaftlichen Arbeitsprozesse auf den einzelnen Gewannen unter allen Landbesitzern abgestimmt und ausgehandelt werden. Damit existierte permanent ein intensiver Diskurs innerhalb dieser Kommunikationsgemeinschaft,

vgl. https://www.leibniz-gwzo.de/de/forschung/mensch-und-umwelt/landschaftsarchaeologie/cervenische-burgen (22.03.2021). Hierzu neben weiteren Publikationen, insbesondere die Bände der Reihe „U źródeł Europy Środkowo-Wschodniej“/,Frühzeit Ostmitteleuropa“ (Salamon, Wołoszyn u. a., 2012; Florek und Wołoszyn, 2016; Wołoszyn, 2017; 2018).

${ }^{6}$ Das Attribut ,traditionell“" ist hierbei nicht negativ gemeint. Diese Bearbeitung bildet für vielfältige linguistische und extralinguistische Analysen eine unverzichtbare Grundlage und ist deswegen nicht zu entbehren. Daneben sind andere Zugänge zu den Eigennamen, die erst in jüngerer Zeit von der Forschung aufgegriffen oder entwickelt wurden, von gleicher Bedeutung. 
der ohne verbindliche Benennungen der einzelnen Gewanne und weiterer Objekte auf der Gemarkung nicht möglich gewesen wäre. Dies ist der pragmatische Kontext, in dem die meisten traditionellen Flurnamen entstanden sind. Diese sind nur dann richtig zu verstehen, wenn dieser Kontext auch berücksichtigt wird. Prinzipiell ist dieser Umstand allgemein bekannt (vgl. z. B. Bauer, 1965, S. $257-$ 263; Šrámek, 2010, S. 128), bei enzyklopädisch-etymologisch ausgerichteten Flurnamenforschungen spielt er jedoch eine eher geringe Rolle.

Besonders wertvolle Quellen bieten diesbezüglich die Agrarreformen des 19. Jahrhunderts, die zu einer Neuvermessung und -verteilung der landwirtschaftlichen Flächen nahezu aller Siedlungen führten. In den früheren preußischen Provinzen werden diese Vorgänge als Separationen bezeichnet ${ }^{7}$, sie erfolgten im Zuge der Modernisierungen hin zur kapitalistischen Wirtschaftsweise aber beinahe überall in Mitteleuropa (Vgl. z. B. Schäfer, 2010). Um die Rechtmäßigkeit der neu eingeteilten Flächen nachzuweisen, wurde die alte Feldeinteilung vermessen und in detaillierten Karten dargestellt. Heute sind diese Separationskarten mitsamt ihren zugehörigen Aktenbeständen (Rezesse) hervorragende Quellen sowohl für die historisch-genetische Siedlungsforschung als auch die Flurnamenforschung. In ihrem Detailreichtum bieten sie tiefe Einblicke in die dörfliche Kommunikationsgemeinschaft, zeigen auf, was benannt wurde und was nicht, ob Benennungen fest sind oder Varianten aufweisen und welche Arten der individualisierenden Bezeichnung gegenüber anderen bevorzugt wurden.

Dieser Blick auf die Details lässt erkennen, dass der Flurnamenschatz nicht nur eine bestimmte Menge einzelner Onyme ist, sondern ein Knäuel von Beziehungen zwischen den vielen Namen, von Konzepten, Analogien, Nachbenennungen und spezifisch proprialen Bedeutungen, die klein- oder großräumige Reichweiten aufweisen können. Eine größere Zahl von Einzeluntersuchungen (Zschieschang, 2003, S. 131-237; 2005; 2011a; 2011b; 2020) ergibt hierbei ein aussagekräftiges und fundiertes Gesamtbild. Zudem ist zu postulieren, dass viele weitere Forschungsrichtungen über Flurnamen - neben lexikografischen ist hier z. B. auch an kognitivistische Perspektiven zu denken - erst durch die gründliche Berücksichtigung der spezifischen Umstände der einzelnen Benennungen zuverlässig werden können.

Auch wenn solche lokal bezogenen Untersuchungen besonders zweckmäßig sind, soll es in diesem Beitrag jedoch nicht vorrangig darum gehen. Denn nicht

7 Wenn auch zum Teil größere Verluste durch den Zweiten Weltkrieg zu beklagen sind (vgl. Anm. 10), sind diese Quellenkomplexe in den Landesarchiven noch zum großen Teil erhalten. Für gewöhnlich finden sie sich in der Tektonik der einschlägigen Archive unter den Provinzialbehörden im Bestand „Generalkommission“ oder „Landeskulturämter“ — auch für die heute zu Polen gehörenden Gebiete. 
immer ist es möglich, den ideal erscheinenden Weg zu beschreiten. Ortsbezogene Studien für alle Gemarkungen einer größeren Landschaft anzufertigen, wäre überdies derart aufwändig, dass es praktisch als beinahe unmöglich bezeichnet werden muss. Demgegenüber gilt es, vorhandene Potenziale und Möglichkeiten möglichst optimal zu nutzen.

Seit einiger Zeit ist es ein Ziel des sorbischen Instituts, die sorbischen Namen als Teil der sorbischen Kultur zu dokumentieren und auf eine zeitgemäße Weise zu präsentieren. Dies kann auf einem umfangreichen Forschungsstand aufbauen, der in mehr als einem Dreivierteljahrhundert intensiver onomastischer Arbeit insbesondere in Leipzig und in Berlin erarbeitet wurde ${ }^{8}$. Damit existieren hervorragende Grundlagen für die Präsentation sorbischer Orts- und Personennamen in zeitgemäßen und öffentlichkeitswirksamen Medien'. Die Dokumentation des sorbischen Namenschatzes weist jedoch auch große Lücken auf. Dies betrifft neben Gewässernamen vor allem Flurnamen. Für diese Namenklasse ist der Forschungsstand geprägt von zahlreichen Einzeluntersuchungen, die sich typologisieren lassen in:

1. Regionale namenkundliche Arbeiten für Teilbereiche der Nieder- und Oberlausitz. Sie sind unterschiedlichen Alters, heterogen strukturiert, sprachwissenschaftlich aber unter Berücksichtigung ihrer Entstehungszeit solide (Muka, 1918; Schwela, 1958; Hoffmann, 1959; Hein, 1963; Alexander, 1965; Sperber, 1967; Gansleweit, 1982).

2. Ortsbezogene Bearbeitungen einzelner Gemarkungen, die unter den verschiedensten Perspektiven entstanden. Sie sind (2a) historisch-heimatkundlich orientiert (z. B. Krüger, 1911/1913; Lehmann, 1979), (2b) siedlungsgenetisch (z. B. Bönisch, 1960; August, 1973; Bönisch und Krausch, 1998) oder auch (2c) namenkundlich (z. B. Gansleweit, 1978; Gansleweit, 2006). Entsprechend unterschiedlich ist auch der Umgang mit den jeweiligen Flurnamen — teilweise werden

${ }^{8}$ Die Leistungen dieser Forschungszentren durch eine Literaturauswahl in einer Fußnote zu würdigen, ist unmöglich und würde den Umfang dieses Beitrags sprengen. Somit seien nur in alphabetischer Reihenfolge die Forscherinnen und Forscher genannt, die wichtige Beiträge für die Lausitz und ihre unmittelbaren Nachbarregionen vorgelegt haben, wobei die Verdienste namentlich nicht Genannter nicht geschmälert sein sollen: Inge Bily, Ernst Eichler, Reinhard E. Fischer, Elżbieta Foster, Klaus-Dieter Gansleweit, Volkmar Hellfritzsch, Karlheinz Hengst, Bernd Koenitz, Klaus Müller, Horst Naumann, Gerhard Schlimpert, Hans Walther, Sophie Wauer, Walter Wenzel, Cornelia Willich, Teodolius Witkowski. Größtenteils sind ihre Werke in der internationalen Onomastik bekannt und leicht zu recherchieren.

${ }^{9} \mathrm{Vgl}$. hierzu https://www.dolnoserbski.de/mjenja/ (22.03.2021); sowie eine Sendereihe im sorbischen Radioprogramm des Rundfunks Berlin-Brandenburg, in der in zweiwöchentlichem Rhythmus jeweils ein sorbischer Name erklärt wird; zeitweise verfügbar unter https://www.rbb-online.de/radio/ sorbisches_programm/Sprachbeitraege/beitragsarchiv-awdioteka.html (22.03.2021). 
sie nur kommentarlos aufgelistet, teils kurz erklärt, aber auch umfassend sprachwissenschaftlich analysiert.

3. Sammlungen in der wissenschaftlichen und heimatkundlichen, auch sorbischen Literatur, in denen Flurnamen lediglich aufgelistet werden (Rostok, 1887; Kühnel, 1890-1899; Gander, 1912; Jordan-Slepjanski, 1913; Kuba-Słoḿeński, 1913). Nähere Angaben zur Lage, zum Charakter oder zur Etymologie sind knapp gehalten oder fehlen gänzlich, so dass der Wert dieser Sammlungen begrenzt bleibt. Eine etymologisch ausgerichtete Kompilation ist hingegen Muka (1927, 1929).

4. Ungedruckte Namensammlungen, die durch verschiedenen Initiativen vom späten 19. bis zur ersten Hälfte des 20. Jahrhunderts entstanden und hier nicht genauer vorgestellt werden können (Brandenburgische Flurnamensammlung; Sächsische Flurnamensammlung). Sie stützen sich auf die Angaben von kundigen Gewährspersonen in den bearbeiteten Orten, in den meisten Fällen der Dorfschullehrer. Als Quellen sind diese Bestände von großer Bedeutung. Weniger bekannt, wenngleich in einschlägigen Werken berücksichtigt (Alexander, 1965; Hein, 1963), blieb eine umfängliche Sammlung von Flurnamen für das sorbische Sprachgebiet, die im Rahmen eines Forschungsprojekts in den Jahren 1954 bis 1958 von Ernst Tschernick/Arnošt Černik zusammengetragen und im Sorbischen Kulturarchiv aufbewahrt wird (Nachlass Černik; vgl. Tschernik, 1958).

Die geschilderte Heterogenität hat auch terminologische Konsequenzen: Die gedruckten Werke sind Literatur, ihrer Funktion nach werden sie jedoch wie auch die ungedruckten Sammlungen für weitere Forschungen als Quellen verwendet. Dies sind sie aber nur bedingt, da sie selbst aus den eigentlichen (Primär-) Quellen schöpfen, also allenfalls Sekundärquellen sind. Nach wissenschaftlichen Kriterien wären natürlich unbedingt die Primärquellen heranzuziehen, was aber in der Praxis aufgrund des großen damit verbundenen Aufwands (von physischen Quellenverlusten ganz zu schweigen ${ }^{10}$ ), nicht immer möglich sein wird. In dieser komplexen Situation erscheint es angebracht, die genannten Werke als Sammlungen zu bezeichnen. Hiermit wird ihr Charakter, dass sie Material für die übergreifende Untersuchung der Namen bereitstellen, am besten verdeutlicht. Gleichzeitig bleibt diese Bezeichnung in Bezug auf ihre inhaltliche Qualität neutral.

Trotz ihrer großen Zahl umfassen diese Sammlungen bei weitem nicht alle Flurnamen für Nieder- und Oberlausitz. In geografischer Hinsicht bleiben größere Lücken, indem einerseits gedruckte Sammlungen (Anstrich 1-3) nicht für alle Teilgebiete existieren, und andererseits auch die ungedruckten Sammlungen (Anstrich 4) nicht für alle Siedlungen vorliegen. Außerdem wurden bisher noch

${ }^{10} \mathrm{Zu}$ den gravierenden Kriegsverlusten insbesondere bei den oben genannten Separationskarten vgl. Bönisch und Krausch (1998, S. 265). 
nicht alle vorhandenen archivalischen Quellen berücksichtigt, so existieren z. B. nicht wenige detaillierte Flurkarten aus dem 18. und 19. Jahrhundert.

Interessant sind hierbei auch kulturgeschichtliche Aspekte. Die meisten der aufgelisteten Sammlungen beruhen - ohne dass hier auf Einzelheiten eingegangen werden kann - auf den Angaben von Gewährpersonen in den einzelnen Orten. Eine besondere Rolle spielten hierbei die Dorfschullehrer, die mit den lokalen Gegebenheiten ebenso vertraut waren wie mit den Grundprinzipien wissenschaftlichen Arbeitens. Zumeist lieferten sie schriftliche Namenlisten ab. Arnošt Černik hingegen besuchte alle Ortschaften persönlich und befragte dort Personen, die Flurnamen kannten.

Fast alle Sammlungsaktivitäten erfolgten in der ersten Hälfte des 20. Jahrhunderts, genauer von etwa 1910 bis 1960, also in weniger als einem Menschenalter. Immerhin gab es in der Umgebung von Cottbus, also im Kern des niedersorbischen Sprachgebietes, gleich drei Gelegenheiten, um Flurnamensammlungen zu unterstützen: in den dreißiger Jahren die Brandenburgische Flurnamensammlung, die Sammlungen von Bogumił Šwjela etwa zur gleichen Zeit (Schwela, 1958; vgl. oben) sowie 20 Jahre später Arnošt Černik (Nachlass Černik; Tschernik, 1958). Damit konnten dieselben Personen theoretisch an mehreren Sammlungen mitgewirkt haben, wobei die Frage besteht, ob sie dies taten. Immerhin galten die sorabistischen Aktivitäten des evangelischen Pfarrers und Sprachwissenschaftlers Bogumił Šwjela im Nationalsozialismus als staatsfeindlich (Schurmann, 2020). Dies könnte manchen potenziellen Partner von einer Mitwirkung abgehalten haben, zumal die Lehrer Staatsbeamte und als solche zur Loyalität verpflichtet waren.

Im Allgemeinen gehen die Gewährpersonen mit niedersorbischen Flurnamen kundig und souverän um, es gibt jedoch auch Mitteilungen wie diese: „Herrn Kreisschulrat Walter, Cottbus, zur Meldung, dass die gewünschte Aufstellung der hiesigen Flurnamen meinerseits nicht erfolgen kann, da dieselben rein wendische Namen sind, die ich weder sprechen, noch schreiben, noch übersetzen kann. Meine diesbezüglichen Bemühungen, besonders betr. des Übersetzens, bei hiesigen Einwohnern blieben ebenfalls erfolglos. " ${ }^{\text {"11 }} \mathrm{Ob}$ in Fällen wie diesem die fehlenden Sprachkenntnisse nur aus Bequemlichkeit vorgeschoben oder tatsächlich gegeben waren, und inwieweit sie im Kontext der nicht nur in der Zeit des Nationalsozialismus verbreiteten Wendenfeindlichkeit der deutschsprachigen Bewohner der Lausitz stehen, wäre noch zu klären. Hier dürfte ein reicher Fundus von direkten oder indirekten Zeugnissen über den damaligen Status des Niedersorbischen vorliegen: Wie die Dorfschullehrer und andere Gewährsleute

${ }^{11}$ So der örtliche Lehrer Müer in: Brandenburgische Flurnamensammlung, Kreis Cottbus, Nr. 2738 Tauer, 19.2.1936. 
mit dieser Sprache umgingen, inwieweit sie sie beherrschten, welches Interesse sie ihr entgegenbrachten, zeigt sich auch in der Qualität ihrer Namensammlungen. Entsprechende Untersuchungen ließen sich evtl. soweit treiben, dass unterschiedliche Schreibweisen häufig auftretender Namen als Indikatoren größerer oder geringerer Sprachkompetenzen gelten könnten.

Auch heute gibt es vor allem in der Niederlausitz zahlreiche ehrenamtliche Flurnamenforscher, die Namen zusammentragen und dabei auch die genannten Sammlungen nutzen. Sie sind in der Region und untereinander gut vernetzt, sowie z. T. auch in Projekte des Sorbischen Instituts eingebunden, in denen es um Dokumentationen des Kulturerbes für Teilbereiche des sorbischen Sprach- und Siedlungsgebietes geht ${ }^{12}$.

Insgesamt liegt damit eine Situation vor, die Flurnamenforschung begünstigt: zahlreiche Vorarbeiten und ein großes Interesse in der Bevölkerung getragen durch ehrenamtliche oder bürgerwissenschaftliche Flurnamenforscher (gegenwärtig oft als ,citizen scientists“ oder lokale Experten bezeichnet). Die Heterogenität der bisherigen Sammlungen, die sich relativ leicht in eine homogene Datenbasis transferieren zu lassen scheint, ist dabei als Vorteil zu sehen, da sie unterschiedliche Perspektiven im Umfang mit Flurnamen repräsentiert.

Andererseits bringt sie ein besonderes Problem mit sich, das hier nur angerissen werden kann. Auch hierbei handelt es sich eigentlich mehr um eine Chance als um ein Problem: Wenn nämlich die Flurnamen bestimmter Orte in mehreren Sammlungen erfasst wurden, ergibt sich die Möglichkeit, die Qualität dieser Sammlungen miteinander zu vergleichen und eine Validierung vorzunehmen. Solche Überlappungen sind sehr häufig und recht komplex, da nicht wenige Ortschaften von gleich drei Sammlungen berücksichtigt wurden. Dies betrifft insbesondere die historische Mitte des niedersorbischen Sprachgebietes, wo - wie oben bereits ausgeführt - für das Bearbeitungsgebiet von Schwela (1958) zwei weitere Sammlungen (Brandenburgische Flurnamensammlung; Nachlass Černik; vgl. Tschernik, 1958) vorliegen.

Dabei wäre zu erwarten, dass bei gründlicher Sammlung weitgehend die gleichen Namen aufgelistet werden, die Divergenzen also gering bleiben. Angesichts des geringen zeitlichen Abstands der Sammlungstätigkeiten und der möglichen personellen Identität der lokalen Sammler ist auch nur in geringem Umfang von zeitbedingten Veränderungen des Flurnamenbestandes auszugehen. Die Realität

${ }_{12}$ Im Modul Niederlausitz: „Zeugnisse der Lebens- und Baukultur der Niederlausitzer Sorben/ Wenden“ des Projekts „Inwertsetzung des Immateriellen Kulturerbes im deutsch-slawischen Kontext (IKE)“, vgl. https://www.serbski-institut.de/de/Zeugnisse-der-Lebens--und-Baukultur-der-Niederlausitzer-SorbenWenden-Drittmittelprojekt/ (22.03.2021). 
sieht jedoch anders aus: Stichproben erbrachten erhebliche Unterschiede zwischen den Namenbeständen, für die sich bislang nur schwer eine Erklärung finden lässt.

Dies sei hier anhand eines einfachen Beispiels, der Namen in der Gemarkung Byhlen, nso. Bělin, nördlich des Spreewalds, illustriert. Hierfür listen sowohl die Brandenburgische Flurnamensammlung als auch die Sammlung von Arnošt Černik zahlreiche Namen auf:

\begin{tabular}{|c|c|c|}
\hline $\begin{array}{c}\text { Černik } \\
\text { deutscher Name }\end{array}$ & $\begin{array}{c}\text { Černik } \\
\text { niedersorbischer Name }\end{array}$ & BFNS \\
\hline Alte Pferdebahn & & Alte Pferdebahn \\
\hline \multirow[t]{2}{*}{ Alter Teich } & Stary gat & Alter Teich \\
\hline & & Am Gitter \\
\hline Bänkchen & Ławka & Bänkchen \\
\hline Bastens Laug & Ług & \\
\hline \multicolumn{3}{|l|}{ Baumschule } \\
\hline \multirow[t]{2}{*}{ Benniks Fichten } & & Bennings Fichten \\
\hline & & Berglang \\
\hline \multicolumn{3}{|l|}{ Bierweg } \\
\hline \multirow[t]{2}{*}{ Birken-Luch } & Brjazowy lug & \\
\hline & & Blabkens Berg \\
\hline Byhlena & Bělena & Byhlena, Bilena \\
\hline \multirow[t]{2}{*}{ Byhlener See } & Bělinski jazor & \\
\hline & & Bullwiese \\
\hline \multirow[t]{5}{*}{ Cottbuser Berg } & Měšćanska góra & \\
\hline & & Ditzk \\
\hline & Dot & Doll \\
\hline & & Vor Dorf \\
\hline & & In Dorf \\
\hline \multicolumn{3}{|l|}{ Drei Grenzen } \\
\hline \multirow[t]{4}{*}{ Drusche-See } & Druže & \\
\hline & Dubrawy & \\
\hline & & Fischeracker \\
\hline & & Fuhrmannstraße \\
\hline \multicolumn{3}{|l|}{ Gahlens Berg } \\
\hline & Gaj & Gai \\
\hline \multicolumn{3}{|l|}{ Goldener Strauch } \\
\hline & & Graulwegchen \\
\hline & & Groblitzka \\
\hline
\end{tabular}




\begin{tabular}{|c|c|c|}
\hline Gr. Zehme-See & Wjeliki zemski jazor & \\
\hline Großes Luch & Wjeliki tug & \\
\hline Guste Lug & Gusty tug & \\
\hline \multicolumn{3}{|l|}{ Hollkens Berge } \\
\hline & & Jurkens Ann sein Kreuz \\
\hline Kauens Laug & Ług & \\
\hline Kauer-See & Kataŕ & \\
\hline \multicolumn{3}{|l|}{ Klausch } \\
\hline Kl. Zehme-See & Mały zemski jazor & \\
\hline Am Kosackenwege & & Kosackenweg \\
\hline \multicolumn{3}{|l|}{ Lehm-Berg } \\
\hline Luschk & $Ł u z ̌ k$ & Luschk \\
\hline Lutzischa & Łucyšća & \\
\hline \multirow[t]{2}{*}{ Im Malin } & Malin & Malin \\
\hline & & Minske Doll \\
\hline Mutzkotte Laug & Muckojty tug & \\
\hline Nassenz & Njaseńc & \\
\hline \multirow[t]{2}{*}{ Neuer Teich } & Nowy gat & Neuer Teich \\
\hline & & Obische \\
\hline \multicolumn{3}{|l|}{ Ochsenknechts Seite } \\
\hline & & Piatzische [nic: Pintzische] \\
\hline Pintschens Berg & Wótšow & \\
\hline Pintschens Quell & & Pintschens Quell \\
\hline Plonitz & Plonica & \\
\hline Ratzens Laug & Ług & \\
\hline \multirow[t]{2}{*}{ Reinscher Laug } & Ług & \\
\hline & & Rücklang \\
\hline \multirow[t]{3}{*}{ Sage-Laug } & Žagty tug & \\
\hline & & Sagrodde \\
\hline & & Schinderkaite \\
\hline Schmulpiatz & Šmulpjac & Smulnipiatz \\
\hline Skopsluch & Eug & \\
\hline Tenken-Berge & Teńki & \\
\hline Theerofen-See & Maznik-jazor & \\
\hline Ugring-See & Hugrink & \\
\hline Winitzke Berge & Winicke góry & \\
\hline \multirow[t]{2}{*}{ Wolfsluch } & Eug & Wolfslauch \\
\hline & & Wulschina \\
\hline Zehmische Berge & Zemske gory & \\
\hline
\end{tabular}


Da es hier nur um die Verdeutlichung der Unterschiede zwischen den Sammlungen geht, erfolgte die Darstellung in vereinfachter Form in alphabetischer Reihenfolge (abweichend von der originalen Anordnung). Zusätzliche Angaben, insbesondere zur Lage, wurden hier ebenso weggelassen wie der stereotype Zusatz zu allen Namen in der BFNS, dass sie aus dem „Volksmund“ stammen, also nicht aus Schriftquellen eruiert wurden. Natürlich wirft diese knappe Auflistung über die Feststellung der Unterschiede zwischen den Sammlungen hinaus zahlreiche weitere Fragen auf, insbesondere zu dem Verhältnis der niedersorbischen Namenformen zu den deutschen Pendants bei Černik und zu deren Authentizität. Diesbezügliche Analysen wären jedoch verfrüht, weil die Aufbereitung der Flurnamensammlungen erst am Anfang steht.

Hier bleibt nur festzustellen, dass neben 14 Namen, die in beiden Sammlungen enthalten sind, mehr als 50 - also fast viermal so viele! — in einer von beiden fehlen. Dabei handelt es sich nicht um einen Ausnahmefall; vielmehr eher um ein Verhältnis, wie es auch in anderen Gemarkungen anzutreffen ist (vgl. auch Zschieschang, 2021). Die Gründe sind momentan noch schwer anzugeben. Einige dieser Bildungen (vor Dorf, in Dorf, Bullwiese, Schinderkaite) sind sprachlich recht belanglos und mögen deshalb nicht beachtet worden sein oder tatsächlich in den wenigen Jahrzehnten, die zwischen den beiden Sammlungen liegen, verschwunden oder neu entstanden sein. Vielleicht überschritt Černik in seiner Betrachtung auch die Grenzen der eigentlichen Gemarkung. Dennoch entgingen ihm einige explizit sorbische Namen.

Dies ist, insbesondere in einem größeren Kontext, noch genauer zu bearbeiten. Momentan ist aber eine Konsequenz entscheidend: angesichts dieser Divergenzen wäre eine bloße Addition verschiedener Namensammlungen nicht sachgerecht. Die Angabe, woher der einzelne Name stammt, ist wesentlich, und die Unterschiede müssen möglichst genau dokumentiert werden, damit sie bei späteren, eingehenden Forschungen auch nachvollzogen werden können. Dann kann jede Gemarkung diesbezüglich individuell untersucht werden, wobei sich aus einer größeren Zahl solcher Studien ein aussagekräftiges Gesamtbild ergibt. Dies schließt den Kreis zum Plädoyer für lokale Untersuchungen am Beginn dieses Beitrags. Für solche und natürlich auch anderweitig ausgerichtete Flurnamenforschungen gilt es momentan auf der Basis der vorhandenen Sammlungen solide und zeitgemäße Grundlagen zu schaffen. Die Voraussetzungen sind dabei gegeben, dies nicht nur für lokale Experten (bzw. citizen scientists) zu tun, sondern gemeinsam mit ihnen. 


\section{UNGEDRUCKTE QUELLEN}

Brandenburgische Flurnamensammlung (Geheimes Staatsarchiv Preußischer Kulturbesitz Berlin, X. HA Brandenburg, Rep. 16 Kleine Erwerbungen).

Nachlass Černik (Sorbisches Kulturarchiv, XXXII Nachlass Arnošt Černik/Ernst Tschernik (19101988) — Projekt „Bevölkerungsstatistik der sorbischen Lausitz“1954-1958).

Sächsische Flurnamensammlung (Sächsisches Hauptstaatsarchiv Dresden, Bestand 13403 Sächsische Flurnamenstelle, 1. Flurnamenforschung, 1.3. Verzeichnisse).

Tschernik, E. (1958). Ausführlicher Abschlussbericht zur Forschungsarbeit, Typoskript, Sorbisches Kulturarchiv, ISL XXXII 22 D.

\section{LITERATUR}

Alex ander, G. (1965). Die sorbischen Flurnamen des Kreises Bautzen (westlich der Spree). Leipzig. Phil. Diss.

August, O. (1973). Beitrag zu den Fragen des hochmittelalterlichen Ausbaus von Tornow. In J. Herrmann (Hrsg.), Die germanischen und slawischen Siedlungen und das mittelalterliche Dorf von Tornow, Kr. Calau (= Schriften zur Ur- und Frühgeschichte, 26) (S. 337-357+ Beilage 7). Berlin: Akademie-Verlag.

Bauer, G. (1965). Flurnamengebung als Feldgliederung. Ein kritischer Beitrag zur Methode der Flurnamenstatistik. In R. Schützeichel und M. Zender (Hrsg.), Namenforschung. Festschrift für Adolf Bach zum 75. Geburtstag am 31. Januar 1965 (S. 245-263). Heidelberg: Universitätsverlag Winter.

Bönisch, F. (1960). Die Fluren der Gemarkung Klein-Räschen vor Ausführung der Gemeinheitsteilung. Jahrbuch für Brandenburgische Landesgeschichte, 11, 101-117.

Bönisch, F. und Krausch, H.-D. (1998). Grießen im Spree-Neiße-Kreis nach der Flurkarte von 1774. Veröffentlichungen des Brandenburgischen Landesmuseums für Ur-und Frühgeschichte, $32,265-290$.

Čīšangs, K. [= Zschieschang, Ch.] (2018). Ūdensdzirnavu nosaukumi Oderas apkārtnē. Ieskats Leipcigas Humanitāro zinātñu centra „Vēsture un kultūra Centrāleiropas austrumu daḷā“ onomastiskajā darbā [Names of Water Mills in the Oder River Basin. An insight into onomastic work at the GWZO in Leipzig]. Onomastica Lettica, 5, 75-94.

Czopek, B. (1984). Apelatyw majdan w toponimii polskiej [Appellative majdan in Polish toponymy]. Onomastica, 29, 69-89.

Czopek, B. (1988). Nazwy miejscowe dawnej ziemi chetmskiej i betskiej (w granicach dzisiejszego państwa Polskiego) [The Settlement Names of the Former Chełm and Bełz Lands (Within the Borders of Present-Day Poland)]. Wrocław-Warszawa-Kraków: Ossolineum.

Florek, M., \& Wołoszyn, M. (Hrsg.). (2016). Wczesnośredniowieczny zespół osadniczy w Czermnie w świetle wyników badań dawnych (do 2010). Podstawy źródlowe [The Early Medieval Settlement Complex at Czermno in the Light of Results from Past Research (up to 2010). Material Evidence]. (2 Bde.). Kraków-Leipzig-Rzeszów-Warszawa: Leipziger Universitätsverlag-Instytut Archeologii i Etnologii PAN-Instytut Archeologii URz.

Ganslew eit, K. D. (1978). Die Flurnamen von Atterwasch. Gubener Heimatkalender, 22, 59-69.

Gansleweit, K. D. (1982). Untersuchungen zur Namenkunde und Siedlungsgeschichte der nordöstlichen Niederlausitz. Die Flur- und Ortsnamen im Bereich des früheren Stiftes Neuzelle (= Deutsch-Slawische Forschungen zur Namenkunde und Siedlungsgeschichte, 34). Berlin: Akademie-Verlag. 
Gansleweit, K. D. (2006). Ergebnisse sprachkundlicher Untersuchungen des Flurnamenguts. In D. Karg und F. Schopper (Hrsg.), Horno - zur Kulturgeschichte eines Niederlausitzer Dorfes (Bd. 2, S. 483-500). Wünsdorf: Brandenburgisches Landesamt für Denkmalpflege und Archäologisches Landesmuseum.

Hein, W. (1963). Die sorbischen Flurnamen des Kreises Bautzen (östlich der Spree). Leipzig. Phil. Diss.

Hoffmann, L. (1959). Die slawischen Flurnamen des Kreises Löbau (= Deutsch-Slawische Forschungen zur Namenkunde und Siedlungsgeschichte, 9). Halle/Saale: VEB Max Niemeyer Verlag.

Jordan-Slepjanski, H. (1913). Dozběrka k lež. mjenam Grodkowskeho wokrjesa [Gleanings (i. e. supplements) to the minor place names of the district Grodk/Spremberg]. Časopis Maćicy Serbskeje, 14, 123-126. https://www.serbski-institut.de/de/dnlarchiv/cms-1913.2107.pdf

Krüger, W. (1912/1913). Die Flurnamen des Stadtgebietes Luckau. Niederlausitzer Mitteilungen, $12,218-251$.

Kuba-Słoḿeński, [...]. (1913). Ležownostne mjena Grodkowskeho wokrjesa [Minor place names of the district Grodk/Spremberg]. Časopis Maćicy Serbskeje, 14, 97-103. https://www.serbskiinstitut.de/de/dnlarchiv/cms-1913.2107.pdf

Kühnel, P. (1890-1899). Die slavischen Orts- und Flurnamen der Oberlausitz. Neues Lausitzisches Magazin, 66, 209-261; 67, 43-126; 69, 1-48 und 257-283; 70, 57-99; 71, 241-288; 73, 125-179; 74, 193-271; 75, 169-253. Neudruck (1982) hrsg. von E. Eichler. Leipzig: Zentralantiquariat der DDR.

Lehmann, R. (1979). Verzeichnis der Flurnamen und Örtlichkeitsbezeichnungen im Senftenberger Stadtgebiet vor der Industrialisierung (nebst 2 Karten). In R. Lehmann (Hrsg.), Quellen zur Geschichte der Niederlausitz (Bd. 3, S. 185-244). Köln: Böhlau Verlag.

Maříková, M., \& Zschieschang, Ch. (Hrsg.). (2015). Wassermühlen und Wassernutzung im mittelalterlichen Ostmitteleuropa (= Forschungen zur Geschichte und Kultur des östlichen Mitteleuropa, 50). Stuttgart: Franz Steiner Verlag.

Mielzarek, Ch., \& Zschieschang, Ch. (Hrsg.). (2019). Usus aquarum. Interdisziplinäre Studien zur Nutzung und Bedeutung von Gewässern im Mittelalter (= Forschungen zur Geschichte und Kultur des östlichen Mitteleuropa, 54). Wien-Köln-Weimar: Böhlau Verlag. https://www.vrelibrary.de/isbn/9783412500870

Muka, A. (1918/1984). Bausteine zur Heimatkunde des Luckauer Kreises. Luckau: Verlag des Kreisausschusses des Luckauer Kreises. Teilweiser Neudruck in: E. Mucke/A. Muka, Abhandlungen und Beiträge zur sorbischen Namenkunde (1881-1929), hrsg. von E. Eichler (S. 399-482). Köln-Wien: Böhlau Verlag.

Muka, E. [= Muka, A.] (1927-1929/1984). Serbske ležownostne mjena a jich woznam [Sorbische Flurnamen und ihre Bedeutung]. Slavia Occidentalis, 6, 225-253; 8, 83-156. Neudruck in: E. Mucke/A. Muka (1984). Abhandlungen und Beiträge zur sorbischen Namenkunde (18811929), hrsg. von E. Eichler (S. 295-398). Köln-Wien: Böhlau Verlag.

Rostok, M. (1887). Ležownostne mjena [Minor Place Names]. Časopis Maćicy Serbskeje, 40, 3-50. https://www.serbski-institut.de/de/dnlarchiv/cms-1913.2107.pdf

Rymar, E., \& Czopek, B. (1993). Nazwy młynów i osad młyńskich na terenie dawnej Nowej Marchii [Names of mills and mill settlements in the area of the former New March]. Onomastica Slavogermanica, 18, 61-115.

Salamon, M., \& Wołoszyn, M. et al. (Hrsg.). (2012). Rome, Constantinople and Newly-Converted Europe: Archaeological and Historical Evidence. 2 Bde. Kraków-Leipzig-Rzeszów-Warszawa: Leipziger Universitätsverlag-Instytut Archeologii i Etnologii PAN-Instytut Archeologii URz.

Schäfer, B. (2010). Das lineare Dorf oder die landschaftsräumliche Revolution. Von der Wandlung einer Kulturlandschaft im 19. Jahrhundert im Einzugsgebiet der Kamienna in Polen. In B. Perlich, 
\& G. van Tussenbroek (Hrsg.), Mittelalterliche Architektur. Bau und Umbau, Reparatur und Transformation. Festschrift für Johannes Cramer zum 60. Geburtstag (S. 219-234). Petersberg: Michael Imhof Verlag.

Schurmann, P. (2020). Bogumil Šwjela und sein politisches Vermächtnis. Zwischen Loyalität und kritischer Distanz. Lètopis, 67, 4-20.

Schwela, Ch. G. (1958). Die Flurnamen des Kreises Cottbus (= Veröffentlichungen des Instituts für Slawistik, 17). Berlin: Akademie-Verlag. Neudruck (2019) Bautzen: Domowina-Verlag.

Sperber, W. (1967). Die sorbischen Flurnamen des Kreises Kamenz (Ostteil) (= Deutsch-Slawische Forschungen zur Namenkunde und Siedlungsgeschichte 18). Berlin: Akademie-Verlag.

Šrámek, R. (2010). Zur Typologie der Flurnamen. In H. Bergmann, \& P. Jordan (Hrsg.), Geographische Namen - Vielfalt und Norm. 40 Jahre institutionalisierte Ortsnamenforschung und -standardisierung in Österreich. 65. Geburtstag von Isolde Hausner. Akten des Internationalen Symposions Wien, 10. und 11. März 2009 (S. 125-135). Wien: Praesens Verlag.

Wołoszyn, M. (Hrsg.). (2017). From Cherven'Towns to Curzon line. The lands on the Middle Bug during the Middle Ages and the historiographic perspective on the formation of Poland's eastern border, $18^{\text {th }}-21^{\text {st }}$ cc. $=$ Od Grodów Czerwieńskich do linii Curzona. Dzieje środkowego Pobuża $w$ wiekach średnich oraz postrzeganie formowania się wschodniej granicy Polski w historiografii XVIII-XXI w., 2 Bde. Kraków-Leipzig-Rzeszów-Warszawa: Leipziger UniversitätsverlagInstytut Archeologii i Etnologii PAN/Instytut Archeologii UR.

Wołoszyn, M. (Hrsg.). (2018). The Medieval Settlement Complex at Gródek upon the Bug River in the Light of Results from Past Research (1952-1955). Material Evidence. Kraków-LeipzigRzeszów-Warszawa: Leipziger Universitätsverlag-Instytut Archeologii i Etnologii PAN/ Instytut Archeologii UR.

Zschieschang, Ch. (2003). „Das Land tuget gar nichts. “ Slaven und Deutsche zwischen Elbe und Dübener Heide aus namenkundlicher Sicht (= Namenkundliche Informationen, Beiheft 22). Leipzig: Universitätsverlag.

Zschieschang, Ch. (2005). Flurnamen als Indikatoren hochmittelalterlicher Siedlung — der Raum um Wittenberg. In A. Burkhardt, U. Föllner, \& S. Luther (Hrsg.), Magdeburger Namenlandschaft. Onomastische Analysen zu Stadt und Region in Geschichte und Gegenwart (S. 187-209). Frankfurt am Main: Peter Lang.

Zschieschang, Ch. (2011a). Flurnamenforschung und Flurforschung - eine Symbiose? In W.-A. Frhr. von Reitzenstein (Hrsg.), Flurnamen, Straßennamen. Jahrespreise 2006, 2007, 2008 der ,Henning-Kaufmann-Stiftung zur Förderung der deutschen Namenforschung auf sprachgeschichtlicher Grundlage (S. 139-180). Hildesheim-Zürich-New York: Georg Olms Verlag.

Zschieschang, Ch. (2011b). Der letzte Rest. Zur Benennung peripherer Flächen auf dörflichen Gemarkungen. In E. Meineke und H. Tiefenbach (Hrsg.), Mikrotoponyme. Jenaer Symposion 1. und 2. Oktober 2009 (S. 325-349). Heidelberg: Universitätsverlag Winter.

Zschieschang, Ch. (2014). Was macht die Namenkunde am GWZO? Mitropa. Jahresheft des GWZO, 2014, 61-63. https://www.leibniz-gwzo.de/sites/default/files/dateien/PDF_Mitropa_2014.pdf

Zschieschang, Ch. (2015a). Zur Benennung von Mühlen im Mittelalter. In M. Maříková, \& Ch. Zschieschang (Hrsg.), Wassermühlen und Wassernutzung im mittelalterlichen Ostmitteleuropa (S. 193-232). Stuttgart: Franz Steiner Verlag.

Zschieschang, Ch. (2015b). Zur Rolle von Flurnamen in der Kulturlandschaft und der Kulturlandschaftsforschung. In B. Aehnlich, \& E. Meineke (Hrsg.), Namen und Kulturlandschaften (= Onomastica Lipsiensia, 10) (S. 375-397). Leipzig: Universitätsverlag.

Zschieschang, Ch. (2017). Das früh- und hochmittelalterliche Siedlungsumfeld von Trepcza, Czermno und Gródek im Lichte der Toponomastik. Eine methodische und areale Standortbestimmung = Osadnictwo wokół Trepcza, Czermno i Gródka we wczesnym średniowieczu okiem językoznawcy. Uwagi metodyczne i analiza przestrzenna. In: M. Wołoszyn (Hrsg.), 
From Cherven'Towns to Curzon Line. The Lands on the Middle Bug During the Middle Ages and the Historiographic Perspective on the Formation of Poland's Eastern Border, the $18^{\text {th }}-21^{\text {st }}$ Century $=$ Od Grodów Czerwieńskich do linii Curzona. Dzieje środkowego Pobuża w wiekach średnich oraz postrzeganie formowania się wschodniej granicy Polski w historiografii XVIIIXXI wieku (S. 161-227). Kraków-Leipzig-Rzeszów-Warszawa: Leipziger UniversitätsverlagInstytut Archeologii i Etnologii PAN/Instytut Archeologii UR.

Zschieschang, Ch. (2018). Zur Entstehung von Mühlennamen. Ein Beispiel aus dem mittleren Odergebiet. In R. Heuser, \& M. Schmuck (Hrsg.), Sonstige Namenarten. Stiefkinder der Onomastik (S. 69-98). Berlin-New York: de Gruyter.

Zschieschang, Ch. (2019). Badania toponomastyczne okolic Grodów Czerwieńskich we wczesnym średniowieczu [Toponomastic research in the surrondings of the Cherven' Towns in the early and High Middle Ages]. In A. Chomová, J. Krško, \& I. Valentová (Hrsg.), Konvergencie a divergencie v propriálnej sfére. 20. slovenská onomastická konferencia Banská Bystrica 26.-28. júna 2017. Zbornik referátov [Convergence and Divergence in the Proprial Sphere. Proceedings of the 20 $0^{\text {th }}$ Slovak Onomastic Conference. Banská Bystrica 26-28 June 2017 (S. 151-163). Bratislava: VEDA.

Zschieschang, Ch. (2020). Die Mark Schmelz in der Dübener Heide. Ein Exempel in Sachen Flurnamenforschung. Namenkundliche Informationen, 112, 419-450. https://nbn-resolving. org/urn:nbn:de:bsz:15-qucosa2-758150

Zschieschang, Ch. (2021). Die Erforschung sorbischer Flurnamen in der Niederlausitz. Forschungsstand und Perspektiven. Namenkundliche Informationen, 113, in print.

\section{SUMMARY}

PERSPECTIVE OF THE RESEARCH ON MICROTOPONYMS IN SORBIAN LUSATIA

When it comes to investigating minor place names, there is a broad range of methods available, reflecting the heterogeneous nature of this class of proper names. Probably the most suitable approach is to examine microtoponyms from the angle of an individual settlement within its boundaries, since it is home to a specific communication community. Seen from this perspective, it becomes apparent that instead of merely a set of individual names, microtoponyms represent a tangle of relations between these names as well as of concepts and analogies, etc.

To obtain an extensive picture of this tangle, as many sources as possible need to be considered. When presenting minor place names as part of Sorbian cultural heritage, a specific situation applies: the high number of different regional and local collections. Many of them have been published: some cover large parts of Lusatia, while others are confined to individual settlements; some of them are onomastic studies with extensive analyses, others are merely uncommented lists of names. Furthermore, there are several handwritten collections in the archives comprising thousands of names. Substantial parts of the study areas overlap. Although one might expect the same names to be found in these different collections, there are in fact remarkable divergencies, as demonstrated here by an example. All in all, Lusatia offers diverse material for research on minor place names. Moreover, the fact that many people are still interested in these names presents a good opportunity for citizen science, which could and should play an important role in future research.

Keywords: minor place-names, name collections, Sorbian cultural heritage, citizen science 